thin film of suitable insulating material coating both surfaces, the metal disk serving as the 'signal plate'. Positive charges are built up on the surface of the insulator by secondary electron emission or possibly by bombardment-induced conductivity. With such a device the quantum efficiency should be roo times that of a photographic plate, the number of significant photo-events which can be recorded per unit area should be at least roo times that possible with fast photographic plates, and the uniform background can be subtracted electronically from the wanted picture in order to enhance its intelligibility.

\title{
9. JOINT COMMITTEE ON IMAGE TUBES
}

\section{By J. S. HALl, United States Naval Observatory}

In December I953 a joint committee on image tubes was established by Dr Vannevar Bush, president of the Carnegie Institution of Washington, and funds were made available for its activities by the Carnegie Corporation of New York. The joint committee includes Merle A. Tuve (chairman) of the Carnegie Institution, L. Marton of the U.S. National Bureau of Standards, W. A. Baum of Mount Wilson and Palomar Observatories, and J. S. Hall of the U.S. Naval Observatory.

During I954 the committee investigated the feasibility of adapting or modifying commercial image tubes for astronomical use, particularly image orthicons and vidicons. Most of this work was carried out at the National Bureau of Standards by L. Marton and E. S. Dayhoff. Except for certain limited applications involving high surface brightnesses, such as the observation of planets or the solar spectrum, it was concluded that the image orthicon in its present commercial form would require several fundamental modifications or improvements to meet the low-brightness requirements of primary interest to this committee. Two types of improvement would be essential. One of these is concerned with the electronic integration and storage of an image for a sufficiently long time without loss of definition; the second concerns the present difficulty of transferring the electronically stored image to a permanently recorded picture without serious loss of useful information. Work on electronic storage tubes is being carried out at several laboratories. Although this work will be watched with considerable interest by the committee, it was decided that a new project in this field was not justified and that the efforts and resources of the committee should be turned to a channel of more immediate promise.

There are two general methods by which the advantage of photocathodes over photographic plates can be utilized, the advantage being that which results from a Ioo-fold gain in quantum efficiency. In paper no. 3 these were designated as methods $(a)$ and $(b)$; they are represented respectively by the converter systems which Prof. Lallemand described in paper no. I and by the electron-storage tubes (such as the image orthicon) which Dr Morton described in paper no. 2. In principle, the two methods offer identical opportunities for an improvement over unaided photography, but in practice some of the image-converter schemes appear to have more immediate promise for low light-level applications entailing long exposures.

During the first half of 1955 the committee launched an effort to develop a particular type of image converter for commercial mass production. This device, which has been explored in the laboratory by Hiltner and Burns, is called a 'thin-film converter'. It has a very thin film stretched across the end where the fluorescent screen would otherwise be located. The photo-electron beam can pass right through this film, but gas molecules cannot; consequently, the thin film permits the electronic image to be formed outside the tube while the high-quality vacuum inside the tube is preserved. When a photographic plate is placed behind the thin film, the impinging electrons create a latent image just as in Lallemand's system.

The Farnsworth Electronics Company and the Radio Corporation of America are both co-operating with the committee in the experimental production and testing of these new 
tubes. The present model is sketched in Fig. 6 . It is about $\mathrm{x} 2 \mathrm{~cm}$. long and comes complete with photocathode, electron optics, and thin film. Since the thin film would be ruptured by atmospheric pressure, the tube is supplied during manufacture with an evacuated glass cap covering the rear end. A spare stock of tubes can thus be kept on the shelf until needed.

When a tube is put into operation, it is installed in a vacuum plate changer. This device provides a rough vacuum around the rear end of the tube so that the protective glass cap can be safely removed from behind the thin film; it also provides a means for getting photographic plates in and out of the system without breaking the vacuum. It is hoped that this technique will eventually enable astronomers to obtain plates with image tubes in a fashion as routine as that already achieved in electron microscopy.

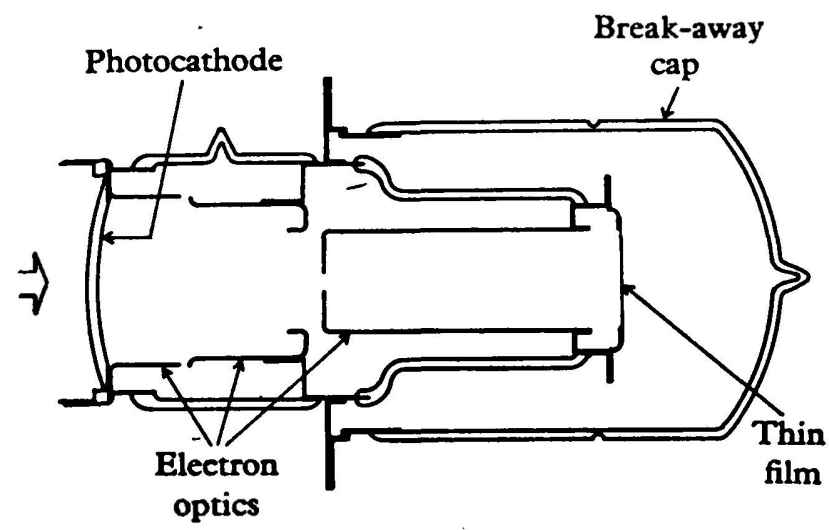

Fig. 6. An image converter of the type which utilizes a very thin film to prevent gases exuded by a photographic emulsion from reaching the photocathode. The operation of this tube is explained in the text. Tubes of this design are being made experimentally by R.C.A. and by the Farnsworth Electronics Company under special contracts with the Joint Committee, whose aim is to evolve a design suitable for commercial mass production.

The primary remaining problem concerns finding the best procedure for producing thin films having optimum characteristics. Many experimental films have been made by Dayhoff at the Bureau of Standards and by Sharpless at the Naval Observatory, and similar exploration is now being carried out by Ford at the University of Virginia. The present specifications require that the thin film be self-supporting across a circular aperture Io $\mathrm{mm}$. in diameter, that it withstand a differential pressure of $10^{-5}$ atmosphere, that the gas leakage through it be negligible, that attenuation of the electrons be less than Io $\mathrm{kV}$., that the loss of resolution due to electron scattering in the film be minimized, and that the film be undamaged by the baking process required in commercial tube production.

As a second line of attack, an attempt is also being made to develop a special type of thin-film converter capable of withstanding full atmospheric pressure, thereby avoiding the complications of the vacuum plate changer described above. In this case the aperture covered by the thin film can be only a narrow slot, but such a slot would be sufficient for many spectroscopic applications. A film of dural $700 \AA$ thick was found to withstand atmospheric pressure when mounted across a slot $0.1 \mathrm{~mm}$. wide by $\mathrm{I} 2 \mathrm{~mm}$. long. Although the scattering of $\mathrm{I} 5 \mathrm{kV}$. electrons by this film was two or three times the desirable maximum, these preliminary results have convinced the committee that there is a possibility of using a thin-film converter for spectroscopy under atmospheric pressure. 